\title{
¿Técnicos o profesionales? ¿Qué opinan los implicados? El caso de los agentes de empleo y desarrollo local (AEDL)
}

\section{Ricard Calvo Palomares}

Universitat de València. Departament de Sociologia i Antropologia Social ricardo.calvo@uv.es

\section{Resumen}

Desde que aparece una actividad laboral —empleo u ocupación- hasta que llega a ser considerada una profesión, muchas son las dificultades que ésta ha de superar. En el presente texto, centramos nuestro interés en analizar las dificultades existentes en una de las actividades surgidas en las últimas décadas del siglo XX - la de generar empleo y desarrollo a nivel local o, lo que es lo mismo, la actividad de los AEDL (agentes de empleo y desarrollo local) - en su proceso de reconocimiento como profesión. En base a lo que se expondrá a lo largo de este artículo, buscaremos conocer la percepción que tiene el propio colectivo de afectados sobre la realidad de su situación profesional, así como de sus posibilidades de convertirse en profesión. Para ello, recurrimos a las informaciones procedentes de una investigación realizada recientemente (2008-2011) desde el Departamento de Sociología y Antropología Social de la Universidad de Valencia. Concretamente, utilizaremos los resultados obtenidos en la realización de 7 grupos de discusión que reunieron a 43 técnicos en desarrollo local de la Comunidad Valenciana.

Palabras clave: agentes de empleo y desarrollo; profesiones; trabajo técnico; desarrollo local; empleo.

\section{Abstract. Technical or professional? What is the opinion of the people involved? The case of employment and local development agents}

From the time a new labour activity arises - a job or an occupation-until it comes to be regarded as a profession, there are many difficulties that must be overcome. In this paper, we analyse the difficulties of a labour activity that emerged in the last decades of the twentieth century aimed at creating employment and promoting local development, namely that of employment and local development agents (AEDL), and the process to recognise this activity as a profession. We seek to determine the perceptions of AEDLs, the reality of their professional situation, and the chances of becoming a profession. To do so, we use data from a recent research study conducted from 2008-2011 by the Department of Sociology and Social Anthropology at the University of Valencia. Specifically, we use the results of seven discussion groups bringing together 43 local development agents in the region of Valencia.

Keywords: employment and development agents; professions; technical work; local development; work. 


\title{
Sumario
}

$\begin{aligned} \text { 1. Introducción: los AEDL, } & \text { 4. Los resultados más destacados } \\ \text { una realidad sobre el territorio } & \text { 5. Conclusiones finales } \\ \text { sión o empleo: el eterno debate } & \text { Referencias bibliográficas } \\ \text { la sociología de las profesiones } & \end{aligned}$

3. La cuestión de estudio y la metodología seguida

\begin{abstract}
A pesar de críticas y contradicciones o de retos insuperables, los ciudadanos en las sociedades contemporáneas desarrolladas o en desarrollo, desean y aspiran, en términos sociológicos, a convertirse en profesionales.
\end{abstract}

(Hughes, 1960: 37)

\section{Introducción: los AEDL, una realidad sobre el territorio}

Tomamos como caso de estudio lo ocurrido con los AEDL en sus 25 años de existencia. Aparecidos en el año 1986, pretendían, como objetivo principal, ser el instrumento que reactivara la economía y creara el nivel de empleo necesario en cada territorio que posibilitara salir de la crisis en la que se encontraba nuestro país en aquellos momentos. Como una manifestación más de las nonatas políticas activas de empleo (en adelante PAE), propusieron un modelo de intervención pública a través del fomento de la creación de puestos de trabajo - por cuenta propia o ajena-, buscando la adecuación de la mano de obra a las necesidades de las empresas, básicamente a través de la formación, y fomentando el ajuste entre oferta y demanda de trabajo por medio de la orientación y la facilitación de información respecto al mercado de trabajo mediante los servicios de empleo —o las entidades colaboradoras de los mismos (Ruiz Viñals et al., 2004: 124).

Concebidos para la acción, ocuparon un espacio inexistente hasta aquel momento y practicaron una actividad totalmente inédita sobre el territorio, clara herencia de las experiencias llevadas a cabo años atrás en países como Inglaterra, Francia o Italia, que potenciaron el aprovechamiento de los recursos endógenos como el elemento de crecimiento y superación de la crisis. Su alto grado de adaptabilidad a las distintas realidades existentes sobre el territorio, la existencia de suculentas ayudas para su contratación y el proceso de descentralización iniciado años atrás en favor de las administraciones locales (si bien muchas de ellas sin una transferencia competencial formal, como es el caso del empleo y el desarrollo local), se han convertido, en estos 25 años, en los factores determinantes de su continuo proceso de crecimiento y presencia sobre el territorio.

Con el paso del tiempo, esta nueva figura ha ido asumiendo un papel fundamental en la implementación sobre el territorio de las distintas actuaciones vinculadas con el empleo y el desarrollo local. Tanto ha sido así que, en el caso que nos ocupa, el de la Comunidad Valenciana, estimamos que cerca del 70\% 
de las PAE desarrolladas han sido gestionadas directamente por este colectivo. Junto a ello, una presencia manifiesta sobre el territorio. En el caso valenciano, 402 municipios de los 536 existentes cuentan con al menos una unidad de AEDL en sus instalaciones (Calvo, 2011: 110), y la Federación Nacional de Profesionales del Desarrollo Local (FEPRODEL) cifra en unos seis mil los AEDL existentes en 2011 en España. Esta presencia territorial ha conllevado una cobertura directa de la población residente en dicho territorio próxima al 90\% e indirecta de la totalidad de ésta (tasa de cobertura poblacional que compara la población de los municipios que cuentan con un servicio de AEDL con el total de la población de la Comunidad Valenciana en un periodo determinado). Todo ello nos permite poner de manifiesto inicialmente el éxito de esta intervención pública en su objetivo máximo de actuar sobre el binomio formado por el territorio y la población (Calvo, 2011: 17).

\section{Profesión o empleo: el eterno debate de la sociología de las profesiones}

Los AEDL son una más de las muchas actividades laborales —ocupaciones o empleos- originadas en la segunda mitad del siglo XX (junto a trabajadores sociales, diplomados en turismo o informáticos, entre otros) que se han encontrado con muchas y variadas dificultades en su proceso de configuración como realidades de carácter profesional.

Por lo general, la transición de empleo a profesión es compleja y no está exenta de discusión teórica, debido principalmente a la ausencia de unos requisitos definidos a priori y a un proceso evolutivo concretado que permitan la catalogación como profesión de una actividad laboral. Tal y como indican Sánchez y Sáez (2009), los fenómenos de profesionalización no siguen una secuencialidad predeterminada: las diferentes profesiones no se desarrollan a través de los mismos caminos, ni llegan a los mismos resultados.

Pese a ello, lo que sí que parece que el estudio emanado desde la sociología de las profesiones permite entrever es un mínimo consenso en torno a los elementos básicos que pueden resultar constitutivos de una profesión y que, por tanto, nos permitirían diferenciarla de una mera actividad, empleo u ocupación laboral. Fruto de la integración de las distintas aportaciones existentes (Hughes, 1960; Wilensky, 1964; Greenwood, 1966; Estruch y Güell, 1976; Larson, 1977; Hoyle, 1980; Guillén, 1990; Sáez, 2003; Berumen et al., 2005; Sáez, Sánchez y Sánchez, 2009; Sánchez y Sáez, 2009), factores como los que enumeraremos a continuación se convierten, a nuestro entender, en los requisitos necesarios para la profesionalización de una ocupación:

1. El tiempo de experiencia acreditada.

2. La existencia de un cuerpo de conocimientos específicos.

3. Un nivel competencial óptimo.

4. Determinadas técnicas aplicadas en el desarrollo de la actividad.

5. Un número (comunidad) representativo de miembros.

6. Una formación superior (titulación oficial). 
7. Unos principios de actuación que configuren un código ético propio.

8. El reconocimiento de un colegio profesional.

9. El reconocimiento de su utilidad social.

10. Una autopercepción común de la profesión entre sus integrantes.

11. La autonomía y el control sobre su proceso de trabajo.

Todos ellos son elementos que quedan recogidos en la figura siguiente, donde mostramos las relaciones y las interacciones que emanan entre ellos en el proceso de profesionalización de una actividad laboral (ver gráfico 1).

Gráfico 1. De la actividad laboral a la profesión (relaciones entre los factores)

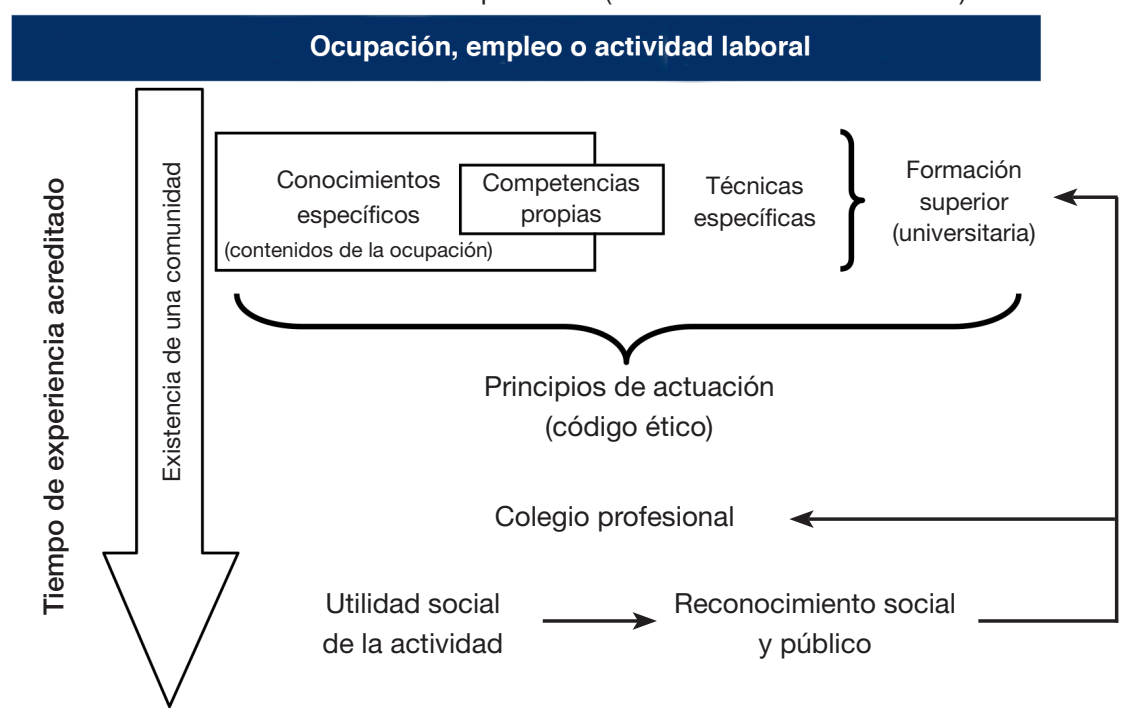

Profesión

Fuente: Calvo, 2011: 231.

\section{La importancia de una percepción común de la profesión entre sus miembros}

Como proceso dinámico que es, la profesionalización de una actividad laboral permite el desarrollo integral de la disciplina de forma consciente, comprometida y organizada, tanto individual como grupalmente, a través de la configuración de los conocimientos, los principios y los valores humanos y sociales presentes en la misma (Moore, 1976: 189). En dicho intento de profesionalizarse, las ocupaciones reformulan la experiencia cotidiana y resignifican la realidad social con la que conviven (Sánchez y Sáez, 2009), estando muy presente la idea de profesionalismo como una actividad que se integra en la 
sociedad, en la que sus actores, como lógica profesional del mismo, convergen e interactúan, como nos indica Freidson (2001), con otras lógicas de la misma, como la burocrática o la ocupacional. Pero, como hemos dicho, el proceso de construcción de la identidad profesional es complejo y no siempre resulta exitoso. Castells (1997: 230-233) sostiene que tan sólo puede hablarse de identidad cuando los actores sociales así lo interiorizan y empiezan a construir su sentido en torno a esa interiorización. Por tanto, no en todos los casos asistiremos con el paso del tiempo a la configuración profesional de una ocupación.

La importancia de que aparezca un discurso profesional consistente, que se diferencie de lo ya existente, que encuentre su espacio social y su utilidad colectiva, se posiciona como fundamental, lo que se materializa en propiciar el desarrollo de una cultura profesional del colectivo y de los grupos pertenecientes a la ocupación. Moore (1976: 189-195) afirma que una profesión puede ser vista a partir de un conjunto de dimensiones estructurales y de actitud, como una ocupación a tiempo integral, caracterizada por la presencia de profesionales con una organización que permite la identificación mutua de intereses comunes y de conocimientos formales y en donde se aceptan las normas y los modelos apropiados para identificarse entre colegas.

Las profesiones están formadas por personas que han superado un período prolongado de educación universitaria, en el que han adquirido no solamente conocimientos técnicos, sino también una orientación vocacional (Guillén, 1990: 76). Moore (1976) precisa que el conocimiento común es indispensable para mantener la unión de un grupo profesional. La identidad profesional se adquiere cuando una persona se integra en un colectivo y adopta no solamente los conocimientos y las habilidades de la profesión en cuestión, sino que, además, adopta los valores y las actitudes que la caracterizan (Vila et al., 1997: 15-19). Por tanto, la idea de proceso es clave, puesto que se concibe la profesión como un evento histórico sujeto a un espacio geográfico y a un contexto concreto que se manifiesta con formas de interés muy diversas en el plano formativo, económico, político, social o cultural (Torstendahl y Burrage, 1990).

La construcción de la identidad profesional resulta de la articulación entre la identidad individual y la identidad colectiva. El proceso de socialización profesional es un proceso de adquisición de identidad conjunta, de desarrollo de sentimientos de pertenencia, y es esencial para desarrollar una identidad profesional consolidada. En el proceso de socialización es donde se integran los conocimientos, las normas, los valores y la cultura de la profesión. Por tanto, se entiende que la sociología de las profesiones, en cada tiempo y lugar, abundará en asuntos y temáticas relacionados con las preocupaciones que surgen en torno a las profesiones y a los profesionales en sus actividades cotidianas (Sáez, Sánchez y Sánchez, 2009).

Por todo ello, a nuestro entender, adquiere una importancia destacada la autopercepción que el propio colectivo tiene de su situación, tanto interna como externa. Y es ahí concretamente donde centramos nuestro debate, en saber cómo perciben los implicados su proceso de profesionalización. Concretamente, aportamos la visión de un colectivo, el de AEDL (agentes de empleo y desarrollo local) que, desde hace unos años, pretenden alcanzar el estatus de profesión. 


\section{La cuestión de estudio y la metodología seguida}

En base a todo lo expuesto, recordamos la hipótesis que planteábamos de manera central en la presente reflexión: ¿existe una percepción compartida en el colectivo de AEDL sobre su imagen como técnicos del desarrollo profesional?, $y$, sobre todo, ¿cómo la perciben?

A fin de responder a esta cuestión, nos basaremos en algunos de los resultados obtenidos en un estudio realizado recientemente en la Comunidad Valenciana (2008-2011), en el que se analizaba la situación en la que se encuentra este colectivo en el momento actual tras más de dos décadas de trabajo. Concretamente, se organizaron siete grupos de discusión de expertos a lo largo de la geografía valenciana que reunieron a 43 profesionales del desarrollo local (38 AEDL pertenecientes a corporaciones locales, 2 AEDL en dependencia de mancomunidades y 3 técnicos de orientación laboral -OPEA-) (ver tabla 1).

La percepción de los implicados la recogemos desde una triple perspectiva, a través del planteamiento de sendas preguntas:

a) ¿Cómo perciben la realidad de su actividad? Por tanto: ¿es su realidad profesional o tan sólo ocupacional?

b) ¿Qué dificultades perciben como existentes en su proceso de profesionalización?

c) Y, a partir de las dos cuestiones anteriores, ¿cuáles son los retos de futuro de la actividad para que pueda ser considerada una profesión?

Nos gustaría señalar que, con carácter previo, en todos los grupos de discusión se realizó una primera ronda de intervenciones individuales sobre cada una de las tres cuestiones planteadas y, además, con respecto a la segunda cuestión planteada, se realizó una dinámica grupal de valoración más o menos consensuada de las distintas dificultades señaladas como respuesta.

Tabla 1. Datos control de los grupos de discusión de expertos

\begin{tabular}{cccccc}
\hline $\begin{array}{c}\text { Código } \\
\text { grupo }\end{array}$ & $\begin{array}{c}\mathbf{N}^{\circ} \text { de } \\
\text { participantes }\end{array}$ & Provincia & $\begin{array}{c}\mathbf{N}^{\circ} \text { de años de } \\
\text { experiencia } \\
\text { media en } \mathrm{DL}\end{array}$ & $\begin{array}{c}\text { Edad media de } \\
\text { los técnicos }\end{array}$ & $\begin{array}{c}\text { Población media } \\
\text { de los técnicos } \\
\text { participantes }\end{array}$ \\
\hline G.1 & 6 & Valencia & 7,4 & 33,4 & \\
G.2 & 7 & Alicante & 10,6 & 35,7 & \\
G.3 & 7 & Castellón & 6,9 & 33,8 & 6.754 \\
G.4 & 6 & Valencia & 12,4 & 29,8 & 13.211 \\
G.5 & 5 & Valencia & 9,7 & 39,7 & \\
G.6 & 5 & Alicante & 8,5 & 35,4 & 32.745 \\
G.7 & 7 & Valencia & 5,8 & 31,1 & \\
\hline
\end{tabular}

Fuente: elaboración propia. 


\section{Los resultados más destacados}

Antes de adentrarnos en el análisis de nuestra cuestión de estudio, cabe recordar que la concepción originaria de los AEDL ya aportaba un grado de indefinición muy grande, si bien no en su definición textual (artículo 7 de la Orden, de 15 de julio de 1999, del Ministerio de Trabajo y Asuntos Sociales, sobre fomento del desarrollo local e impulso de los proyectos y empresas calificadas como $\mathrm{I}+\mathrm{E})$, donde se concretaba que eran trabajadores de las corporaciones locales o de las entidades dependientes o vinculadas a una administración local, que tenían como misión principal colaborar en la promoción y en la implantación de políticas activas de empleo relacionadas con la creación de actividad empresarial, definición que se ha mantenido invariable con el paso de los años, pese a los cambios que la realidad de los técnicos ha sufrido. Donde sí observamos un mayor nivel de indefinición es cuando la misma orden enumeraba, en el artículo siguiente, las funciones a desarrollar por ellos. Son las siguientes:

1. Prospección de recursos ociosos o infrautilizados de proyectos empresariales de promoción económica local e iniciativas innovadoras para la generación de empleo en el ámbito local, identificando nuevas actividades económicas y posibles emprendedores.

2. Difusión y estímulo de oportunidades potenciales de creación de actividad entre los desempleados, los promotores y los emprendedores, así como instituciones colaboradoras.

3. Acompañamiento técnico en la iniciación de proyectos generadores de nuevos empleos para su consolidación en empresas, asesorando e informando sobre la viabilidad técnica, económica y financiera, así como, en general, sobre los planes de lanzamiento de las empresas.

4. Apoyo a los promotores de las empresas, una vez constituidas éstas, acompañando técnicamente a los mismos durante las primeras etapas de funcionamiento, mediante la aplicación de técnicas de consultoría en gestión empresarial y asistencia en los procesos formativos adecuados para coadyuvar a la buena marcha de las empresas creadas.

5. Cualesquiera otras que contribuyan a la promoción e implantación de políticas activas de empleo e impulse la creación de actividad empresarial.

Es, por tanto, una figura cuyo contenido laboral resulta cuanto menos controvertido desde sus inicios. A ella, se le asigna explícitamente la reactivación económica del territorio — el tan ansiado desarrollo — a través del aprovechamiento de sus recursos endógenos, pero también se la acota operativamente, con lo cual se centran sus actuaciones en torno al desarrollo de las políticas activas para el empleo sobre un territorio determinado. Esta característica acompañará toda su existencia, lo cual marcará su evolución posterior, y se convertirá en un factor limitador de su realidad profesional veinticinco años después. Tanto ha sido así que esta controversia entre lo teórico - lo definido o establecido en la norma - y lo real — fruto de la aplicación práctica sobre cada territorio - se ha posicionado como una desventaja no resuelta que ha 
Tabla 2. Análisis DAFO de los 25 años de actividad de los AEDL

\begin{tabular}{ll}
\hline \multicolumn{1}{c}{ Debilidades } & \multicolumn{1}{c}{ Amenazas } \\
\hline - El desconocimiento inicial de lo que es la & - Los orígenes de la actividad (depreciación \\
actividad. & en origen). \\
- El personalismo asumido por la actividad. & - Dependencia económica del puesto (sub- \\
- El clientelismo subvencionado (costum- & vención temporal). \\
brismo). & - Modelo sin objetivos y asistémico. \\
- La dependencia política y la falta de auto- & - Modelo cortoplacista. \\
nomía operativa y decisional. & - Ausencia de una regulación adecuada. \\
- La juventud de la profesión. & - Modelo estático de oferta (repetitivo). \\
- La temporalidad de la actividad (inseguri- & - Tipos y características de los programas. \\
dad laboral). & - Reconocimiento oficial de la actividad (la \\
- La detección de las necesidades del terri- & Administración no les presta atención ni \\
torio. & los tiene en cuenta). \\
- La aparición de localismos (puestos ad & - Sin reconocimiento social. \\
hoc, individualismos localistas). & - No hay un aprendizaje colectivo (sinergia \\
- No han conseguido una autopercepción & profesional). No hay una plataforma donde \\
colectiva común. & compartir sus experiencias. \\
- Continúan sin unos principios de actuación & - Incertidumbre sobre su futuro y rol en \\
(código ético). & el contexto de los ajustes en el ejercicio \\
- No han sabido publicitar (vender) su traba- & de las ofertas públicas (subvenciones, \\
jo a la sociedad. & empleo, etc.). \\
No existe una entidad que represente
\end{tabular}

- No existe una entidad que represente «realmente» al colectivo.

\begin{tabular}{ll}
\hline \multicolumn{1}{c}{ Fortalezas } & \multicolumn{1}{c}{ Oportunidades } \\
\hline - Modelo autoconfigurado, experiencial. & - Modelo público (oportunidad en el sector \\
- Poseen capacidad profesional (aprendizaje). & privado). \\
- Son un número representativo, con pre- & - Pueden ser un referente. \\
- sencia territorial. & - Visión integral de los procesos de desa- \\
- Muchos se han creído su trabajo. & rrollo de un municipio y de los servicios \\
- Alto grado de motivación e implicación & públicos. Saben de lo que hablan. \\
aportado al puesto. & - En muchas ocasiones, no tienen compe- \\
- Crecimiento continuo (en cantidad y en & tencia en su materia sobre el territorio. \\
contenidos) de los programas de empleo & - Prospección de recursos ociosos o infrau- \\
a los programas de desarrollo local. & tilizados y fuentes de financiación e infor- \\
- Son un referente (porcentaje de PAE desa- & mación para proyectos empresariales de \\
rrolladas). & promoción económica local e iniciativas \\
- Proximidad del servicio. & innovadoras para la generación de empleo \\
- Han dado estabilidad al modelo. & en el ámbito local, que identifiquen nuevas \\
- Reconocimiento universitario (formación & actividades y posibilidades emprendedoras. \\
de postgrado). & Acompañamiento técnico en la iniciación \\
- Capacidad de adaptación a los cambios & de proyectos empresariales generadores \\
(¿innovación?). & de nuevos empleos, asesorando e infor- \\
& mando sobre la viabilidad técnica, eco- \\
& nómica y financiera, como también, en \\
& general, sobre planes de lanzamiento de \\
& empresas.
\end{tabular}

Fuente: Calvo y Martínez-Puche, 2012 
dificultado un crecimiento funcional-operativo armonizado a todo el colectivo en los distintos territorios.

Colectivo que, ante la insuficiente regulación inicial de su trabajo, y sobre todo de la manera en que lo debía desarrollar, ha ido improvisando, y por tanto aplicando una metodología totalmente ad hoc — adaptada a las características de cada territorio, de cada entorno existente o incluso de cada persona que ocupaba el puesto- . Pese a que, a priori, cualquier adaptación puede parecer positiva, en este caso, la adaptación máxima enunciada ha supuesto una individualización de los puestos de trabajo de los AEDL, con la consiguiente aparición de una individualización en su autopercepción, apostando por la existencia de tantos perfiles de AEDL como puestos de estos pudieran aparecer sobre el territorio.

Mucho ha influido en ello la inexistencia, en todo este tiempo, de un perfil competencial definido. La no concreción de una serie de funciones, tareas y competencias constitutivas del puesto no ha hecho sino facilitar la aparición de un conflicto de rol en el que han caído estos técnicos en el desarrollo de su actividad. Todos estos elementos inherentes a la esencia de los AEDL no han hecho sino agravar muchos otros, que han ido apareciendo con el paso del tiempo. Factores aparecidos en esta evolución que vemos reflejados en el siguiente cuadro, que nos los presenta clasificados según un análisis DAFO. Es decir, diferenciándolos entre sí, estos se han convertido en una desventaja (debilidad o amenaza) o en una ventaja (fortaleza u oportunidad).

Como podemos comprobar en el cuadro anterior, muchos de los elementos allí recogidos no hacen sino poner de manifiesto la no existencia de una autopercepción común de su realidad como AEDL. Esta cuestión, la visión de los implicados y las posibles connotaciones posteriores, se convierte en el punto central de análisis que presentamos a continuación.

\section{Cuestión 1. Ser técnico AEDL,} ¿es realmente una profesión o es una mera ocupación (empleo)?

Las informaciones recogidas muestran una opinión generalizada de que el puesto de trabajo del técnico AEDL responde a la configuración de una actividad de carácter profesional, y que no tan sólo es un empleo con características ocupacionales. De hecho, en una primera ronda individual de intervenciones realizada en todos los grupos, nueve de cada diez de los participantes así lo afirmaron con rotundidad. Tan sólo tres técnicos se posicionaron en contra, puesto que opinaron al respecto que, a esta ocupación, nunca llegaría a otorgársele un carácter profesional. Pese a estos resultados, cabe mencionar que, de aquellos técnicos que apostaban por la condición de trabajo profesional, más de dos tercios de ellos opinaban que, hoy por hoy, no lo era, y que, si los factores contextuales continuaban como lo estaban actualmente, difícilmente llegaría a configurarse como tal.

Algunos de los comentarios más destacados realizados por los AEDL en los distintos grupos son los que reproducimos a continuación, que no hacen sino ejemplificar la situación descrita: 
Tabla 3. Principales dificultades del proceso de profesionalización (valoración individual)

\begin{tabular}{lr}
\hline Compromiso de la Administración pública & $27,9 \%$ \\
Ausencia de un colegio profesional & $16,3 \%$ \\
Inexistencia de un perfil profesional para el técnico AEDL & $13,9 \%$ \\
Carencia de titulación universitaria & $11,6 \%$ \\
Subvención que regula el puesto & $7,0 \%$ \\
Falta de conciencia colectiva & $4,6 \%$ \\
Otros & $18,6 \%$
\end{tabular}

Fuente: Calvo, 2011.

[...] claramente veo que es una profesión, aunque estamos muy, demasiado, vinculados con la empresa pública y en dependencia de una subvención, y nuestro campo de actuación profesional puede ser mucho más amplio [...] (G.2)

[...] donde yo trabajo lo consideran, al menos hoy por hoy, como una ocupación y no como una profesión, porque no nos ven como tal, no tenemos definidas una tareas, hacemos de todo y tenemos que saber de todo. Somos un verdadero cajón de sastre [...] (G.2)

Es una profesión, pero es muy importante cómo nos considere el resto, y ahí fallamos [...] (G.5)

Debería ser una profesión, pero la percepción que tienen de nuestro trabajo es pobre, muy pobre. Te encuentras en reuniones en que hay técnicos municipales que todavía no saben lo que es un AEDL. (G.1)

Mientras no existan unas tareas definidas, donde se determine nuestro trabajo — como ocurre con muchas otras_-, difícilmente seremos una profesión. (G.1)

Nos falta difusión de lo que es la figura del AEDL, para que nos respeten como técnicos [...] (G.4)

Yo creo que somos una profesión, aunque no hacemos profesión, hacemos ocupación. La orden nos orienta hacia las políticas de empleo, y eso es lo que hacemos. Muchos AEDL nos limitamos a llevar a la práctica cuatro programas de empleo, pero no participamos del verdadero desarrollo local de nuestro municipio. Somos técnicos pero no nos consideran como técnicos [...] en cualquier Ayuntamiento todos saben quién es el arquitecto o el ingeniero en obras públicas y que es lo que hacen, y eso que no se mueven de sus sillas, y sin embargo a nosotros, que nos movemos continuamente, no nos tienen en cuenta para prácticamente nada. (G.7)

\section{Cuestión 2. Dificultades y limitaciones existentes para el proceso} de profesionalización de la actividad del técnico AEDL

Para responder a la segunda de las cuestiones, aquella que entroncaba directamente con las dificultades y las limitaciones del proceso de profesionaliza- 


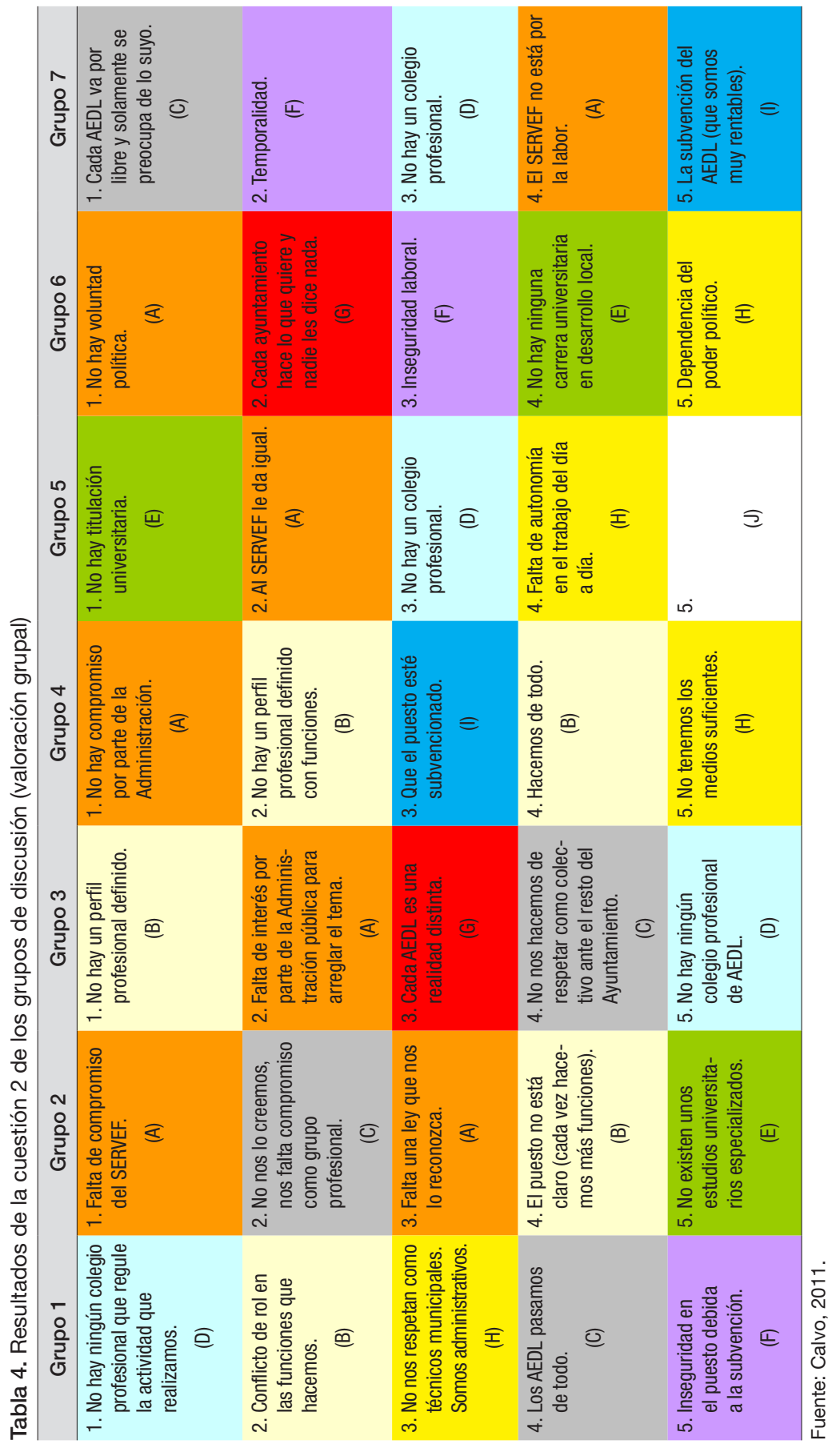


Tabla 5. Principales dificultades del proceso de profesionalización (valoración grupal)

\begin{tabular}{lcc}
\hline \multicolumn{1}{c}{ Dificultad } & Puntuación & $\%$ \\
\hline Compromiso de la Administración pública (A) & 28 & 26,7 \\
Perfil profesional (B) & 17 & 16,2 \\
Concepción como colectivo (C) & 13 & 12,4 \\
Colegio profesional (D) & 12 & 11,4 \\
Titulación universitaria (E) & 8 & 7,6 \\
Temporalidad (F) & 8 & 7,6 \\
Localismo (G) & 7 & 6,7 \\
Respeto como técnicos (H) & 7 & 6,7 \\
Subvención (I) & 4 & 3,8 \\
Otros (J) & 1 & 0,9 \\
Total & 105 & 100 \\
\hline
\end{tabular}

Fuente: Calvo, 2011.

ción, se pidió a los participantes que cada uno de ellos aportara su opinión particular en una primera ronda, y que, además, con posterioridad, entre todos, intentaran generar un resultado grupal más o menos consensuado, estableciendo y priorizando cinco de las dificultades y de las limitaciones aportadas.

Respecto a la valoración colectiva, se presentan a continuación, en la siguiente tabla 3, los resultados conjuntos obtenidos por cada uno de los siete grupos participantes, transcritos con las expresiones utilizadas por cada uno de ellos y tomados por el participante designado como secretario (los distinguimos mediante letras a efectos descriptivos para el lector). Dichos grupos recogen el entramado de dificultades del proceso de profesionalización percibidas por los propios afectados.

Esta representación la podemos simplificar en una única tabla, en la que, una vez ponderados cada uno de estos niveles, podamos ordenarlos según aquellos que han obtenido una puntuación mayor (ponderación realizada a partir de la clasificación realizada en la tabla mediante la asignación de 5 puntos a los factores de primer orden, 4 puntos a los de segundo orden, 3 puntos a los de tercer orden, 2 a los de cuarto orden y 1 a los de quinto orden).

\section{Cuestión 3. Orientaciones y retos de futuro de la actividad. ¿Tiene futuro?} ¿Qué necesita para ser reconocida como tal?

Y, por lo que respecta a la tercera cuestión planteada, la totalidad de los AEDL en desarrollo local participantes en los grupos de discusión coincidió en la perspectiva de considerar que la actividad tiene mucho futuro en sí misma, y con ella también tiene futuro el puesto de trabajo como AEDL. Como argumento principal, se fundamentaron en su condición de necesarios en cualquier contexto económico, sea éste de recesión o de expansión económica. En dicho sentido, representativo de lo anterior es lo señalado por uno de los participantes: 
[...] hoy por hoy, cumplimos con un papel social que ninguna otra actividad municipal puede cumplir [...] (G.3)

También conviene mencionar que prácticamente una tercera parte de los participantes en los diferentes grupos consideró que la actividad no tiene futuro con las características de ejecución con las que se encuentra actualmente, es decir: subvención, temporalidad o funciones no definidas, entre otras.

[...] todo pasa por llegar a ser considerados como tal, si no, no podemos llegar a nada $[\ldots . .].(\mathrm{G} .1)$

[...] pero cómo van a reconocer los demás nuestro trabajo si hay veces que no nos lo creemos ni nosotros y, sobre todo, no nos ponemos de acuerdo [...] (G.7)

A mi entender, los políticos confían poco en nosotros, sólo cuando les conviene [...] necesitamos que confíen en nosotros y nos dejen hacer [...] (G.2)

El SERVEF no quiere saber nada de nosotros. Somos como un hijo tonto, al que se cría pero no se le da demasiada confianza [...] (G.3)

[...] los ayuntamientos nos contrataron porque resultábamos muy baratos y les dábamos un gran servicio. Hacemos de todo [sonríe], pero ahora que tienen que mojarse y plantearse asumirnos, todo son pegas y problemas [...] (G1)

No estamos en igualdad de condiciones con el resto de técnicos municipales. Hacemos mucho más teniendo mucho menos reconocimiento. (G.4)

[...] no tener todavía un colegio profesional que nos represente es un gran freno a nuestro reconocimiento como profesión. (G.5)

\section{Conclusiones finales}

Hay que partir de la realidad de la profesión y no de los sueños de sus profesionales.

(Estruch y Güell, 1976)

Del análisis en profundidad de los discursos desarrollados en cada una de las reuniones, hemos observado la existencia de una serie de elementos apuntados por los participantes que se convierten en determinantes en el proceso de conversión del empleo de AEDL a profesión. Se trata de cinco elementos necesarios para la profesionalización de este colectivo que ponen de manifiesto la importancia que lo externo — el entorno social — tiene para articular adecuadamente este proceso, pero que, sin una actuación paralela a nivel interno, el entorno operativo de la ejecución del trabajo poco futuro tiene. 
Gráfico 2. Variables de futuro de la profesión

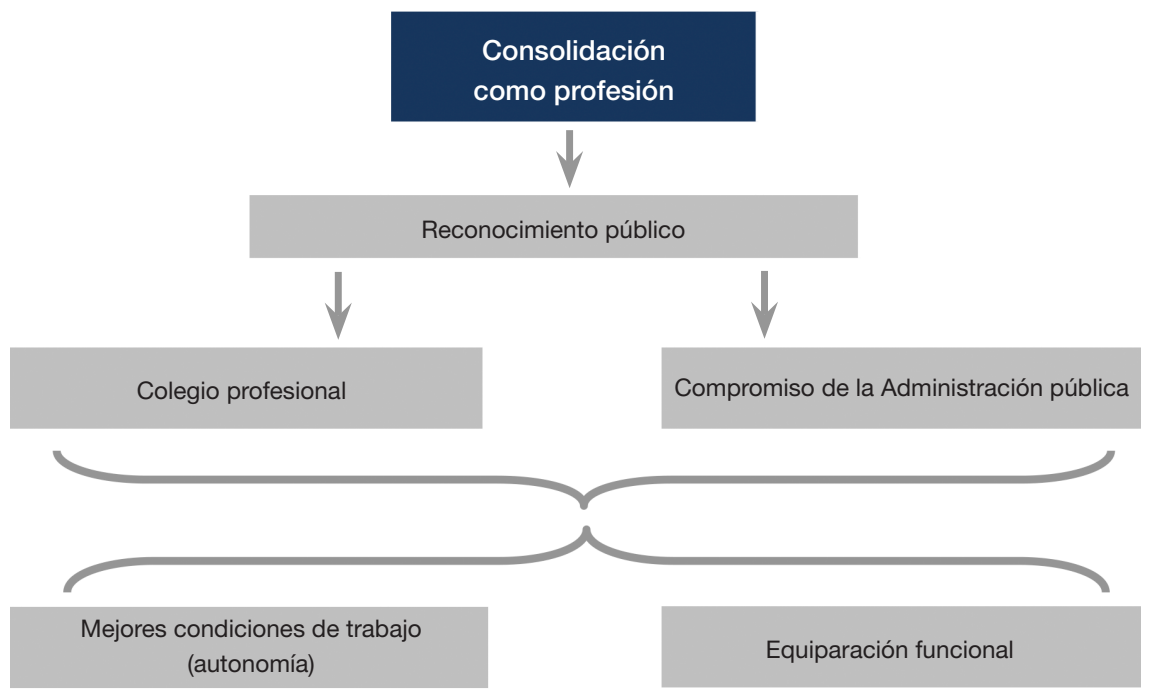

Fuente: Calvo, 2011.

Factores como el reconocimiento público del trabajo realizado, manifestado a partir de la utilidad social otorgada, que, como hemos expuesto, no ha sido una consideración alcanzada o conseguida, así como la existencia de un compromiso de la Administración pública, formal, público, oficial y consecuente, son otros de los elementos no existentes hasta el momento. Una manifestación de este compromiso es la autorización administrativo-oficial para la constitución de un colegio profesional, como el ente de representación colectiva que vele por la adecuada aplicación de un código deontológico - eticoprofesional- y por unas normas reguladoras que protejan a la profesión. Si estos tres factores eran de carácter externo, los dos restantes, es decir, la equiparación funcional y las mejores condiciones de desarrollo del trabajo, constituyen una dimensión interna vinculada con características del desarrollo operativo del puesto. Elementos que, pese a ser internos, tienen una correlación directa con los externos. Representamos los elementos enunciados en el gráfico 2.

A tenor de los resultados obtenidos expuestos más arriba, serían dos las líneas conclusivas básicas que propondríamos, las cuales ponen de manifiesto que:

1. La percepción generalizada por parte del colectivo apunta a que los factores determinantes en su proceso de configuración profesional son, en su mayor parte, de carácter externo al colectivo, y sobre los que en principio poco o nada pueden hacer. 
2. Una percepción mayoritaria de que tienen futuro como profesión, aunque se trata de una perspectiva muy supeditada a la existencia de determinados elementos previos, algunos de los cuales, en un momento como el actual, parecen inalcanzables.

Como cierre, cabe mencionar que también hemos de tener en cuenta que todo ello está muy supeditado al contexto socioeconómico en el que nos encontramos y a la respuesta pública operada, ya que, contextualizando estos resultados a las circunstancias actuales de recortes, apuntamos una situación que puede diferir de la presentada, en la que la percepción de los técnicos cambie completamente y que, por tanto, afecte de manera determinante a su proceso de profesionalización, lo cual podría retrasarlo considerablemente e incluso impedirlo.

\section{Referencias bibliográficas}

Berumen, Nora; Gomar, Silvia y GómeZ, Pedro (2005). Ética del ejercicio profesional. México: CECSA.

BERTILSSON, Margaretha (2003). «Estado de Bienestar, profesiones y ciudadanos». En: SÁeZ, C. J.; SVEnsSOn, L. y SÁnCheZ, M. (coords.) (2003). Sociología de las profesiones, pasado, presente y futuro. Murcia. Diego Marín.

CALVO, Ricard (2011). AEDL (Agente de Empleo y Desarrollo Local): Una aproximación sociológica al estudio de una profesión. Valencia. PUV (Publicacions de la Universitat de València).

Calvo, Ricard y Martínez-PuChe, Antonio (2012). «Empleo y desarrollo desde lo local: un análisis DAFO de 25 años de actividad de los AEDL en la Comunitat Valenciana». En: MARTíneZ-PUCHE, A. y CALVO, R. (2012). Valoraciones técnicas y repercusiones territoriales sobre el ejercicio profesional del desarrollo local: Presente y futuro. Alzira (Valencia): Germania.

CASTELLS, Manuel (1997). La era de la información. Madrid. Alianza Editorial.

EstruCH, Juan y GÜELL, Antonio M. (1976). Sociología de una profesión: Los asistentes sociales. Barcelona: Península.

Freidson, Eliot (2001). Professionalism. The third logic. Cambridge: Polity Press.

GrEENWOOD, Ernest (1966). «Professionalism: The Sociologist's Decoy». En: Work and Occupations, 1, 6-23.

GuilléN, Mauro F. (1990). «Profesionales y burocracia: Desprofesionalización, proletarización y poder profesional en las organizaciones complejas». Revista Española de Investigaciones Sociológicas, 5, 35-52. $<$ http://dx.doi.org/10.2307/40183479>

HoYLE, Eric (1980). Professionalization and desprofessionalization in education. Londres. Kogan Page.

HugHES, Everett C. (1960). "The professions in society». The Canadian Journal of Economics and Political Science, XXVI (1), 19-37.

LARSON, Magali S. (1977). The Rise of Professionalism: A sociological analysis. Berkeley: University of California Press.

MOORE, Wilbert E. (1976). The professions: roles and rules. Nueva York: Russell Sage Foundation. 
Ruiz Viñals, Carmen et al. (2004). Politicas sociolaborales: Un enfoque multidisciplinar. Barcelona: UOC.

SÁEZ, Juan (2003). "Las profesiones sociales: La educación social en perspectiva». En: SÁnCHeZ, M.; SÁez, J. y Svensson, L. Sociología de las profesiones: Pasado, presente y futuro. Murcia: Diego Martín.

SÁEZ, Juan; SÁNCHEZ, Mariano y SÁNCHEZ, Elena (2009). "¿Sociología de las profesiones en España?: Entre la carencia y la necesidad de consolidación». Universitas Tarraconensis: Revista de Ciències de l'Educació, 1, 15-101.

SÁNCHEZ, Mariano y SÁEZ, Juan (2009). «El estudio de las profesiones: la potencialidad del concepto de profesionalización». Universitas Tarraconensis: Revista de Ciències de l'Educació, 1, 103-118.

Torstendahl, Rolf y Burrage, Michael (coords.) (1990). The Formations of Professions. Londres: Sage.

VILA, Bernardo et al. (1997). «Aproximación al contexto actual de enfermería». Cultura de los Cuidados: Revista de Enfermería y Humanidades, 1, 63-69.

WILENSKY, Harold L. (1964). "The professionalization of everyone?». American Journal of Sociology, 70, 137-58.

<http://dx.doi.org/10.1086/223790> 